\section{De Vitae Origine}

The Origins of Life on Earth. By L. E. Orgel. Pp. 237. (Chapman and Hall: London, June 1973.) £3 boards; $£ 1.95$ paper.

Origin and Development of Living Systems. By J. Brooks and G. Shaw. Pp. $\mathrm{xi}+412$. (Academic: London and New York, May 1973.) f5.

THE arrival of the space age has rocketed the interest in the study of the origin of life to new heights. The search for life beyond the Earth, the declared goal of space biology, is intimately associated with the scientifically larger question of the origin of life in the Universe. The Oparin-Haldane hypothesis of chemical evolution has now become the focus of extensive and sophisticated investigations in many laboratories around the world.

The awareness of the general public and the interest evinced by the nonspecialist in science has created a demand for the latest information on the subject in what augurs to be a flood of publications on the beginnings of life. Orgel has written, not for the professional biologist or chemist, but for the freshman in college, the advanced high school student and the general reader, who has a limited knowledge of chemistry and molecular biology. Orgel himself is one of the leading practitioners in the chemistry of the origin of life. A theoretical chemist, who has turned to biochemistry and organic chemistry, he has successfully elucidated a number of chemical pathways presumed to be necessary prerequisites for the emergence of life.

From a discussion of the historical background he gives an account of the history of the Earth's primitive atmosphere and the sources of energy necessary for the synthesis of organic compounds. $\mathrm{He}$ then describes the processes which gave rise to the building blocks of life. Now comes the opportune moment to examine our concept of life, before embarking on a search for life beyond the Earth.

I was disappointed that a good portion of the book has been spent on the background necessary for understanding the problem. I missed the excitement and thrill which I would have expected in picking up a book on the origin of life, written by a scientist actively engaged in its study. But the clarity of presentation, and the thoroughness of the discussion on the critical issues, make this book a valuable addition to the shelves of the general reader interested in an up-to-date and succinct account of current thinking on the problem of life's beginning.

Brooks and Shaw, on the other hand, have approached their study of the origin of life from a very different point of view. Having been involved in the study of pollen grains and their relationship to microspores, they became interested in the ancient Precambrian sediments. These studies led them to the analysis of some of the oldest sediments on Earth. According to their own admission, they felt that there was a great lack of knowledge among chemists, both in the organic and inorganic fields, about the relationship of the geochemical aspect to the problem of the origin of life. Brooks and Shaw present the geochemical background to chemical evolution in readable fashion.

The book starts with an account of the Universe and the evolution of the Earth and its atmosphere. The authors then proceed to the chemicals of life, and finally to an analysis of the Precambrian fossil record and the story of carbonaceous chondrites. Although this book is designed for the nonspecialist and the general reader, a great deal of the material would be of value to the investigator and advanced student.

Of major interest are the chapters dealing with the Precambrian fossil record. Here I was delighted with the note of personal involvement. The excellent pictures provide a refreshing dimension to a stimulating discussion.

The weakness of the book is that the authors, on the one hand, have attempted to cover far too much within a limited number of pages, and, on the other, aimed at too large an audience. If the book was intended for the nonspecialist, some of the chapters, though well written, may be above his grasp.

CYril PonNamperuma

\section{Early quanta}

Quantum Mechanics: New Approaches to Selected Topics. By H. J. Lipkin. Pp. xv +465 . (North Holland: Amsterdam and London; American Elsevier: New York, 1973.) Dfl. 95; \$33.30.

THIs book grew from the lecture notes accrued by the author while giving postgraduate courses on quantum mechanics over a decade both in the United States and in Israel. It differs considerably from many of the standard texts intended for use with such courses. It comprises six monographs on selected topics such as the Mössbauer effect, many body quantum mechanics, Kaon physics, scattering theory, Feynman diagrams and relativistic quantum mechanics. These are presented at a much earlier level than in the conventional texts alongside which they are intended to be read.

The first is based on Dirac's use of the concept of photon polarisation as a general introduction to quantum mechanics. The idea of states of polarised light leads on to the development of a matrix representation, essentially Jones's calculus. There are other texts such as States, Waves and Photons by Simmons and Guttmann which are both more readable and comprehensive in their coverage of this topic. Some reference by the author to Jones and Mueller matrices might be useful as reference.

The second monograph, comprising three chapters, deals with momentum transfer with specific reference to the Mössbauer effect. Here a clear and concise listing is given which includes details of earlier experiments on resonance phenomena. Momentum transfer within a solid matrix, involving phonon interactions and nuclei, is then described with emphasis on beta decay and electron scattering by complex nuclei. This monograph is mainly concerned with the concept of a form factor and requires only an understanding of a free particle and harmonic oscillator in order to be led up to a formal treatment of second quantisation.

The treatment of many particle quantum mechanics follows in the next monograph which deals in turn with fermions and the application of fermion pairs to superconductivity. The concepts of time-dependent perturbation theory are then introduced in the next monograph on the treatment of Kaon decay. A chapter on one-dimensional scattering models provides an excellent introduction to this important part of quantum mechanics and is followed by three chapters dealing with the many body interaction problem. A comprehensive presentation of Thomas-Fermi and Hartree-Foch models is followed by a treatment of elementary excitation processes including grant resonances. The final section in this monograph introduces the reader to spectral and Green's function without entering fully into their applications.

The reader is introduced next to timedependent perturbation theory through Feynman diagrams, using third order 'bremsstrahlung' processes as an example. The polaron is also used as an example of a quantum field approach to solid state physics.

The final monograph introduces the reader to symmetries, invariance and relativistic quantum mechanics. Starting with the Dirac equation, the reader is introduced to gauge transformations, the Lorentz group as a case of symmetry transformation and finally to charge conjugation.

Each monograph in this excellent book is introduced by a summary and ends with a problem test. The book is recommended to graduate students and research physicists looking for a pedestrian but fairly informal treatment of these topics.

D. W. GoodwIN 